VOL. II (1974), 167-180.

\title{
A tauberian theorem related to the modified Hankel transform
}

\section{Kusum Soni and R.P. Soni}

\begin{abstract}
The modified Hankel transform arises naturally in connection with certain semigroup operations on measures in probability theory.
\end{abstract} We give a tauberian theorem for this transform when certain higher moments exist. The probabilistic significance of our result is that it translates a regularity condition on the transform into a direct condition on the measure. This complements earlier results by $\mathrm{Pitman}$ and Bingham for the trigonometric and the modified Hankel transform respectively.

\section{Introduction}

Let $F$ be a probability measure on $[0, \infty)$ and let

$$
\Phi_{v}(x)=\Gamma(v+1) \int_{0}^{\infty}(x t / 2)^{-\nu} J_{v}(x t) d P(t), \quad v \geq-1 / 2 .
$$

Recently, Bingham [4] gave some abelian and tauberian results for the transform defined by (1.1). He proved that if $L(t)$ is a slowly varying function in the sense of Bojanic and Karamata [5] as $t \rightarrow \infty$ and $0<\alpha<2$, then

$$
1-F(t) \sim t^{-\alpha} L(t), t \rightarrow \infty,
$$

if and only if

$$
1-\Phi_{v}(x) \sim x^{\alpha} L(1 / x) 2^{-\alpha} \frac{\Gamma(1+\nu) \Gamma(1-\alpha / 2)}{\Gamma(1+\nu+\alpha / 2)}, x \rightarrow 0 .
$$

Bingham's results are based on those given earlier by Pitman [8] for the Received 17 April 1974. 
cosine transform, $v=-1 / 2$. Bingham and $\mathrm{Pitman}$ discuss these implications at the boundary points, $\alpha=0$ and $\alpha=2$, also. However, for $\alpha>2$, they give only the abelian implication. Our object in this paper is to give the related tauberian result.

\section{Statement of the main result}

If we integrate (1.1) by parts and use the relation

$$
\frac{d}{d t}\left[t^{-v_{J}}(t)\right]=-t^{-v_{J}}{ }_{v+1}(t)
$$

we obtain

$$
G(x)=c x \int_{0}^{\infty}(x t)^{-v_{J}} v_{v+1}(x t) g(t) d t, \quad v \geq-1 / 2
$$

where

$$
G(x)=1-\Phi_{v}(x)
$$

$$
g(t)=1-E(t),
$$

and

$$
c=2^{\nu} \Gamma(v+1)
$$

For $\alpha>2$, the Pitman-Bingham Theorem can be stated as follows.

THEOREM A. If $n \geq 1,2 n<\alpha \leq 2 n+2$, and

$$
\mu_{2 n}=-\int_{0}^{\infty} t^{2 n} d g(t)<\infty,
$$

then

$$
g(t) \sim t^{-\alpha} L(t), \quad t \rightarrow \infty
$$

implies 
(2.8) $G(x)-\sum_{r=1}^{n} \frac{(-1)^{r-1} \Gamma(1+\nu) \mu_{2 r}}{2^{2 r} \Gamma(1+r) \Gamma(1+\nu+r)} x^{2 r}$

$$
\sim \begin{cases}\frac{\Gamma(1+\nu) \Gamma(1-\alpha / 2)}{2^{\alpha} \Gamma(1+\nu+\alpha / 2)} x^{\alpha} L(1 / x), & x \rightarrow 0,2 n<\alpha<2 n+2, \\ \frac{(-1)^{n} \Gamma(1+\nu)}{2^{2 n+1} \Gamma(n+1) \Gamma(n+\nu+2)} x^{2 n+2} \int_{0}^{1 / x} t^{2 n+1} g(t) d t, & x \rightarrow 0, \alpha=2 n+2 .\end{cases}
$$

We prove the following converse.

THEOREM B. Let $n \geq 1,2 n<\alpha \leq 2 n+2$, and let $G(x)$ be the transform of $g(t)$ defined by (2.2). If $g(t)$ is bounded, decreases to zero, and

$$
\int_{0}^{\infty} \operatorname{tg}(t) d t<\infty
$$

then, for some constants $c_{1}, c_{2}, \ldots, c_{n}, c_{n+1}$,

$$
\text { (2.10) } \quad G(x)-\sum_{r=1}^{n} c_{p} x^{2 x} \sim c_{n+1} x^{\alpha} L(1 / x), x \rightarrow 0, c_{n+1} \neq 0,
$$

implies

(2.11) $g(t) \sim c_{n+1} \frac{2^{\alpha} \Gamma(1+\nu+\alpha / 2)}{\Gamma(1+\nu) \Gamma(1-\alpha / 2)} t^{-\alpha} L(t), \quad t \rightarrow \infty, \quad 2 n<\alpha<2 n+2$

or

(2.12) $\int_{0}^{t} u^{2 n+1} g(u) d u \sim c_{n+1} \frac{(-1)^{n} 2^{2 n+1} \Gamma(n+1) \Gamma(n+v+2)}{\Gamma(1+v)} L(t)$,

$$
t \rightarrow \infty, \alpha=2 n+2 .
$$

Furthermore,

(2.13) $\quad c_{r}=\frac{(-1)^{r-1} \Gamma(1+\nu)}{2^{2 r-1} \Gamma(r) \Gamma(1+v+r)} \int_{0}^{\infty} t^{2 r-1} g(t) d t, \quad r=1,2, \ldots, n$.

We note that (2.9) holds if and only if $\mu_{2}$, defined by (2.6), is finite. In what follows, we assume that the slowly varying function $L(x)$ is positive and measurable in $0 \leq x<\infty$. Furthermore, without loss of 
generality, we may also assume that both $L(x)$ and $[L(x)]^{-1}$ are locally bounded.

\section{Proof of Theorem $B$}

We prove the theorem with the help of some lemmas. Let $g(s)$ and $G(s)$ be the Mellin transforms of $g(t)$ and $G(t)$ respectively, that is,

$$
g(s)=\int_{0}^{\infty} t^{s-1} g(t) d t, \quad s=\sigma+i \tau,
$$

and

$$
G(s)=\int_{0}^{\infty} t^{s-1} G(t) d t
$$

The integral (3.1) converges absolutely in $0<0 \leq 2$. Since

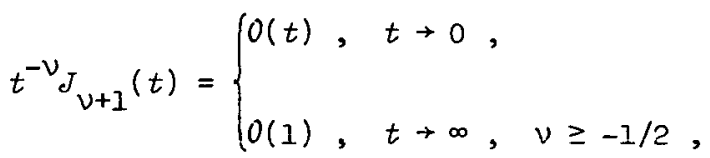

the integral (2.2) converges absolutely, and

$$
G(t)= \begin{cases}O\left(t^{2}\right), & t \rightarrow 0, \\ O(t), & t \rightarrow \infty .\end{cases}
$$

Hence the integral (3.2) converges absolutely in $-2<\sigma<-1$.

LEMMA 1. Under the assumptions of Theorem $\mathrm{B}$, we have

$$
g(s)=\frac{2^{s+\nu} \Gamma(1+v+s / 2)}{c \Gamma(1-s / 2)} G(-s), 1<\sigma<2,
$$

where $c$ is defined by (2.5).

Proof. By the absolute convergence of the double integral in $2<\sigma<3$,

$$
\begin{aligned}
\int_{0}^{X} x^{-s} G(x) d x & =c \int_{0}^{\infty} g(t)\left(\int_{0}^{X}(x t)^{-\nu^{1-s} J_{\nu+1}}(x t) d x\right) d t \\
& =c \int_{0}^{\infty} t^{s-2} g(t)\left(\int_{0}^{X t} u^{1-s-\nu_{J}} v_{\nu+1}(u) d u\right) d t .
\end{aligned}
$$


The inner integral converges absolutely and by $[7$, p. 326, (1)],

$$
\int_{0}^{\infty} u^{1-s-v_{J}} v_{v+1}(u) d u=\frac{2^{1-s-\nu} \Gamma(3 / 2-s / 2)}{\Gamma(v+s / 2+1 / 2)}
$$

Hence, by the dominated convergence theorem,

$$
G(1-s)=\frac{2^{1-s} \Gamma(\nu+1) \Gamma(3 / 2-s / 2)}{\Gamma(\nu+s / 2+1 / 2)} g(s-1), 2<\sigma<3,
$$

which proves (3.5).

Proof of Theorem B (continued). Now we consider the integral

$$
I(x)=\int_{0}^{x} t^{\beta}(x-t)^{\gamma} g(t) d t
$$

where

$$
\begin{gathered}
\beta=2 n+1, \\
\gamma=2 n+4+[\nu],
\end{gathered}
$$

[v] denotes the greatest integer function.

Since

(3.11) $\quad \int_{0}^{x} t^{\beta-s}(x-t)^{\gamma} d t=x^{\beta+\gamma+1-s} \frac{\Gamma(\beta+1-s) \Gamma(1+\gamma)}{\Gamma(\beta+\gamma+2-s)}, \beta>\sigma-1$,

by the Parseval relation for the Mellin transform [13, p. 60],

$$
I(x)=(2 \pi i)^{-1} \int_{\delta-i \infty}^{\delta+i \infty} x^{\beta+\gamma+1-s} \frac{\Gamma(\beta+1-s) \Gamma(\gamma+1)}{\Gamma(\beta+\gamma+2-s)} g(s) d s, 1<\delta<2 .
$$

By $(3.5)$,

$$
I(x)=(2 \pi i)^{-1} \int_{\delta-i \infty}^{\delta+i \infty} x^{\beta+\gamma+1-s} \psi(s) G(-s) d s, 1<\delta<2,
$$

where

$$
\psi(s)=2^{s} \frac{\Gamma(\gamma+1) \Gamma(\beta+1-s) \Gamma(1+\nu+s / 2)}{\Gamma(\nu+1) \Gamma(\beta+\gamma+2-s) \Gamma(1-s / 2)} .
$$

The poles of $\Gamma(1+v+\varepsilon / 2)$ lie in the half plane $\sigma<0$. Therefore, $\psi(s)$ is analytic in $\sigma>0$ except for a finite number of simple poles at $\sigma=2 n+3,2 n+5, \ldots$. By the well known properties of the $\Gamma$-function, 


$$
\begin{aligned}
\frac{\Gamma(1+\nu+s / 2)}{\Gamma(1-s / 2)} & =\pi^{-1} \Gamma(1+\nu+s / 2) \Gamma(s / 2) \sin (\pi s / 2) \\
& =\left(|\tau|^{\sigma+\nu}\right), \quad s=\sigma+i \tau,|\tau| \rightarrow \infty .
\end{aligned}
$$

Thus

$$
\psi(s)=O\left(|\tau|^{\nu+\sigma-\gamma-1}\right), \quad|\tau| \rightarrow \infty .
$$

By (3.12),

$$
I(x)=(2 \pi i)^{-1} \int_{\delta-i \infty}^{\delta+i \infty} x^{\beta+\gamma+1-s} \psi(s)\left(\int_{0}^{\infty} t^{-s-1} G(t) d t\right) d s .
$$

By (3.1.5), the double integral converges absolutely. Hence,

$$
\begin{aligned}
I(x) & =(2 \pi i)^{-1} x^{\beta+\gamma+1} \int_{0}^{\infty} G(t)\left(\int_{\delta-i \infty}^{\delta+i \infty} x^{-s} t^{-s-1} \psi(s) d s\right) d t \\
& =(2 \pi i)^{-1} x^{\beta+\gamma+1} \int_{0}^{\infty} G(u / x)\left(\int_{\delta-i \infty}^{\delta+i \infty} u^{-s-1} \psi(s) d s\right) d u .
\end{aligned}
$$

Let

$$
H(x)=G(x)-\sum_{r=1}^{n} c_{r} x^{2 r}
$$

Our next step is to show that $I(x)$ remains unchanged if $G$ is replaced by $H$ in (3.16).

LEMMA 2.

$$
\begin{aligned}
\int_{0}^{\infty} u^{\mu}\left(\int_{\delta-i \infty}^{\delta+i \infty} u^{-s-1} \psi(s) d s\right) d u & =2 \pi i \psi(\mu), \quad 2 \leq \mu \leq 2 n+2, \\
\psi(2 n+2) & =\lim _{s \rightarrow 2 n+2} \psi(s) .
\end{aligned}
$$

Proof. Let

$$
\phi(u)=\int_{\delta-i \infty}^{\delta+i \infty} u^{\mu-s-1} \psi(s) d s
$$

By $(3.15)$,

$$
\phi(u)=O\left(u^{\mu-\delta-1}\right), u \rightarrow 0, \quad 1<\delta<2 .
$$

Since $\psi(s)$ is analytic in $\delta \leq \operatorname{Re}(s)<2 n+3$, by (3.15) again 


$$
\begin{aligned}
\phi(u) & =\int_{\delta_{1}-i \infty}^{\delta_{1}+i \infty} u^{\mu-s-1} \psi(s) d s, 2 n+2<\delta_{1}<2 n+3 \\
& =O\left(u^{\mu-\delta_{1}-1}\right), \quad u \rightarrow \infty .
\end{aligned}
$$

Thus the repeated integral in (3.18) converges for $\delta<\mu<\delta_{1}$. Obviously,

$$
\text { (3.22) } \begin{aligned}
\int_{0}^{1} \phi(u) d u & =\int_{\delta-i \infty}^{\delta+i \infty}(\mu-s)^{-1} \psi(s) d s \\
& =\int_{\delta_{1}-i \infty}^{\delta_{1}+i \infty}(\mu-s)^{-1} \psi(s) d s+2 \pi i \psi(\mu), \quad 2 \leq \mu \leq 2 n+2 .
\end{aligned}
$$

Also, by shifting the line of integration from $\operatorname{Re}(s)=\delta$ to $\operatorname{Re}(s)=\delta_{1}$,

$$
\begin{aligned}
\int_{1}^{\infty} \phi(u) d u & =\int_{1}^{\infty}\left(\int_{\delta_{1}-i \infty}^{\delta_{1}+i \infty} u^{\mu-s-1} \psi(s) d s\right) d u \\
& =-\int_{\delta_{1}-i \infty}^{\delta_{1}+i_{\infty}}(\mu-s)^{-1} \psi(s) d s, \quad 2 \leq \mu \leq 2 n+2 .
\end{aligned}
$$

Hence, by (3.22) and (3.23),

$$
\int_{0}^{\infty} \phi(u) d u=2 \pi i \psi(\mu), 2 \leq \mu \leq 2 n+2,
$$

which proves the lemma.

For later use, we note the following:

$$
\psi(2 r)=0, r=1,2, \ldots, n,
$$

and

$$
\begin{aligned}
\psi(2 n+2) & =\lim _{s+2 n+2} 2^{2 n+2} \frac{\Gamma(n+v+2) \Gamma(2 n+2-s)}{\Gamma(v+1) \Gamma(1-s / 2)} \\
& =(-1)^{n}{ }^{2 n+1} \frac{\Gamma(n+1) \Gamma(n+v+2)}{\Gamma(v+1)} .
\end{aligned}
$$

We now return to the proof of Theorem B. By Lemma 2 and (3.24), 


$$
\frac{I(x)}{x^{\beta+\gamma+1}}=(2 \pi i)^{-1} \int_{0}^{\infty} H(u / x)\left(\int_{\delta-i \infty}^{\delta+i \infty} u^{-s-1} \psi(s) d s\right) d u,
$$

where $H$ is defined by (3.17). We are interested in the behavior of $I(x)$ as $x \rightarrow \infty$. By $(2.10)$ and $(3.4)$,

$$
|H(u / x)| \leq M(u / x)^{\alpha} L(x / u), 2 n<\alpha \leq 2 n+2,
$$

for some constant $M$. The dominant behavior of $L(x / u), x \rightarrow \infty$, is given by the following lemma. This result is not new and, in a slightly different form, was given by Pitman [8, Lemma 2]. The technique, however, has been used quite often $([3],[5])$.

LEMMA 3. If $L(t)$ is slowly varying, $L(t)$ and $\{L(t)\}^{-1}$ are Zocally bounded, then, for every $n>0$,

$$
\frac{L(x t)}{L(x)}=O\left(t^{-\eta}\right), x \rightarrow \infty,
$$

uniformly in $0<t \leq 1$ and

$$
\frac{L(x t)}{L(x)}=O\left(t^{n}\right), \quad x \rightarrow \infty,
$$

uniformly in $1 \leq t<\infty$.

Proof. Let

$$
L_{1}(x)=x^{-\eta} \sup _{0<t \leq x}\left\{t^{n} L(t)\right\}
$$

and

$$
L_{2}(x)=x^{n} \sup _{t \geq x}\left\{t^{-\eta} L(t)\right\}
$$

$x^{\eta} L_{1}(x)$ is an increasing and $x^{-\eta} L_{2}(x)$ is a decreasing function of $x$. Also, it is known ([1], [2], [5]) that $L_{j}(x) \sim L(x), x \rightarrow \infty, j=1,2$. If $0<t \leq 1$,

$$
(x t)^{\eta} L(x t) \leq(x t)^{n} L_{1}(x t) \leq x^{\eta_{L}} L_{1}(x)
$$

and, if $1 \leq t<\infty$,

$$
\left.(x t)^{-\eta_{L}} L t\right) \leq(x t)^{-\eta_{L_{2}}}(x t) \leq x^{-\eta_{L_{2}}(x)} .
$$


The relations $(3.28)$ and (3.29) follow immediately from the above inequalities.

We return to the consideration of (3.26). By (3.27) and Lemma 3,

$$
\frac{H(u / x)}{x^{-\alpha} L(x)}=O\left(u^{\alpha-\eta}+u^{\alpha+\eta}\right), x \rightarrow \infty,
$$

uniformly in $0<u<\infty$. Choose $n$ such that $\delta<\alpha-\eta<\alpha+\eta<\delta_{1}$. By $(3.20)$ and $(3.21)$, we can apply the dominated convergence theorem to the integral in $(3.26)$. Since, $H(u / x) \sim c_{n+1}(u / x)^{\alpha} L(x)$ pointwise as $x+\infty$, we have

$$
\frac{I(x)}{x^{\beta+\gamma+1-\alpha} L(x)} \sim \frac{c_{n+1}}{2 \pi i} \int_{0}^{\infty} u^{\alpha}\left(\int_{\delta-i \infty}^{\delta+i \infty} u^{-s-1} \psi(s) d s\right) d u
$$

or, by Lemma 2 ,

$$
I(x) \sim c_{n+1} x^{\beta+\gamma+1-\alpha} L(x) \psi(\alpha), \quad x \rightarrow \infty .
$$

It is known ([8, Lemma 3$]$ ) that if $\xi(t)$ is monotone and $\xi_{I}(t)=\int_{0}^{t} u^{p} \xi(u) d u$ is of index $q$ as $t \rightarrow \infty, q>0$, then $t^{p} \xi(t)$ is of index $q-1$. Since $I(x)$ is of index $2 n+2+\gamma-\alpha$, by repeated application of the above result, we see that

$$
h(x)=\int_{0}^{x} t^{2 n+1} g(t) d t
$$

is of index $2 n+2-\alpha$ as $x \rightarrow \infty$. Let

$$
h(x)=x^{2 n+2-\alpha} L^{*}(x), x \geq 1,
$$

so that $L^{*}(x)$ is slowly varying as $x \rightarrow \infty$. For $0 \leq x<1$, define $L^{*}(x)$ to be a locally bounded and integrable function. Then, 


$$
\begin{aligned}
I(x) & =\gamma \int_{0}^{x}(x-t)^{\gamma-1} h(t) d t \\
& =\gamma \int_{0}^{x}(x-t)^{\gamma-1} t^{2 n+2-\alpha} L^{*}(t) d t+\phi_{1}(x) \\
& =\gamma x^{\gamma+2 n+2-\alpha} \int_{0}^{1}(1-u)^{\gamma-1} u^{2 n+2-\alpha_{L} *}(u x) d u+\phi_{1}(x),
\end{aligned}
$$

where $\phi_{1}(x)=O\left(x^{\gamma-1}\right)$ as $x \rightarrow \infty$. To obtain the behavior of the above integral as $x \rightarrow \infty$, we may use a known result [3], or we may use the "dominated convergence" technique which is justified by Lemma 3 . Hence,

$$
I(x) \sim x^{\gamma+2 n+2-\alpha} L^{*}(x) \frac{\Gamma(\gamma+1) \Gamma(2 n+3-\alpha)}{\Gamma(\gamma+2 n+3-\alpha)}, x \rightarrow \infty .
$$

By $(3.30)$,

$$
L^{*}(x)=c_{n+1} L(x) \frac{\Gamma(\gamma+2 n+3-\alpha)}{\Gamma(\gamma+1) \Gamma(2 n+3-\alpha)} \psi(\alpha)
$$

If $\alpha=2 n+2, L^{*}(x)=c_{n+1} L(x) \psi(2 n+2)$, so that by (3.25),

$$
\int_{0}^{x} t^{2 n+1} g(t) d t \sim c_{n+1}(-1)^{n} 2^{2 n+1} \frac{\Gamma(n+1) \Gamma(n+\nu+2)}{\Gamma(\nu+1)} L(x), x \rightarrow \infty,
$$

which is (2.12). If $2 n<\alpha<2 n+2$, by (3.13),

$$
L^{*}(x)=c_{n+1} 2^{\alpha} \frac{\Gamma(1+\nu+\alpha / 2)}{(2 n+2-\alpha) \Gamma(\nu+1) \Gamma(1-\alpha / 2)} L(x) .
$$

Since $h(x)$ is of index $2 n+2-\alpha>0, t^{2 n+1} g(t)$ is of index $2 n+1-\alpha$ as $x \rightarrow \infty$. We now employ reasoning similar to that used earlier to obtain

$$
x^{2 n+1} g(x) \sim c_{n+1} 2^{\alpha} \frac{\Gamma(1+\nu+\alpha / 2)}{\Gamma(\nu+1) \Gamma(1-\alpha / 2)} x^{2 n+1-\alpha} L(x), x+\infty .
$$

This proves (2.11). Finally, we want to prove that the coefficients $c_{p}$ must satisfy (2.13). If $2 n<\alpha<2 n+2$, this follows directly from Theorem A. However, for the case $\alpha=2 n+2$, Theorem $A$ is not applicable since (2.12) does not imply (2.7) even when $g(u)$ is decreasing. The proof of this assertion depends on some results given in [11]. However, (2.13) follows from the following lemma. 
LEMMA 4. Let $n \geq 1$. If $g(t)$ is bounded and decreases to zero as $t \rightarrow \infty$, then (2.12) implies (2.13).

Proof. Since $g(t) \downarrow 0,(2.12)$ implies that

$$
t^{2 n+2} g(t)=o\{L(t)\}, \quad t \rightarrow \infty .
$$

For the sake of convenience, let

$$
k(t)=\Gamma(v+1)(t / 2)^{-v_{J+1}}(t)
$$

and

$$
a_{r}=\frac{(-1)^{r-1} \Gamma(v+1)}{2^{2 r-1} \Gamma(r) \Gamma(v+r+1)},
$$

so that

$$
k(t)=\sum_{r=1}^{\infty} a_{r} t^{2 r-1} .
$$

Now

$$
\begin{aligned}
G(x)-\sum_{r=1}^{n} a_{r} x^{2 r} \int_{0}^{\infty} t^{2 r-1} g(t) d t-a_{n+1} x^{2 n+2} \int_{0}^{1 / x} t^{2 n+1} g(t) d t \\
\quad=x \int_{0}^{\infty}\left\{k(x t)-\sum_{r=1}^{n} a_{r}(x t)^{2 r-1}\right\} g(t) d t-a_{n+1} x^{2 n+2} \int_{0}^{1 / x} t^{2 n+1} g(t) d t \\
=x \int_{0}^{1 / x}\left\{k(x t)-\sum_{r=1}^{n+1} a_{r}(x t)^{2 r-1}\right\} g(t) d t \\
\quad+x \int_{1 / x}^{\infty}\left\{k(x t)-\sum_{r=1}^{n} a_{r}(x t)^{2 r-1}\right\} g(t) d t \\
=I_{1}+I_{2} .
\end{aligned}
$$

We shall prove that $I_{j}=0\left\{x^{2 n+2} L(1 / x)\right\}, x \rightarrow 0, j=1,2$. Since $k(u)$ is bounded, by a known result [3],

$$
\begin{aligned}
\int_{1}^{\infty}\left\{k(u)-\sum_{r=1}^{n} a_{r} u^{2 r-1}\right\} & u^{-2 n-2} L(u / x) d u \\
& \sim L(1 / x) \int_{1}^{\infty}\left\{k(u)-\sum_{r=1}^{n} a_{r} u^{2 r-1}\right\} u^{-2 n-2} d u, \quad x \rightarrow 0 .
\end{aligned}
$$


Therefore, by $(3.34)$,

$$
\begin{aligned}
I_{2} & =o\left\{\int_{I}^{\infty}\left[k(u)-\sum_{x=1}^{n} a_{r} u^{2 r-1}\right](u / x)^{-2 n-2} L(u / x) d u\right\} \\
& =o\left\{x^{2 n+2} L(1 / x)\right\}, x \rightarrow 0 .
\end{aligned}
$$

Next, let $\varepsilon>0$. Choose $0<\delta^{\prime}<1$ such that

$$
\left|k(u)-\sum_{r=1}^{n+1} a_{r^{u^{2}-1}}\right|<\varepsilon u^{2 n+1}, 0<u<\delta^{\prime} .
$$

Furthermore, let

$$
\begin{aligned}
& I_{1}=x\left\{\int_{0}^{\delta^{\prime} / x}\right.\left.+\int_{\delta^{\prime} / x}^{1 / x}\right\}\left\{k(x t)-\sum_{r=1}^{n+1} a_{p}(x t)^{2 r-1}\right) g(t) d t \\
&=I_{3}+I_{4} \\
&\left|I_{3}\right|<\varepsilon x \int_{0}^{\delta^{\prime} / x}(x t)^{2 n+1} g(t) d t \\
&<\varepsilon M_{1} x^{2 n+2} L\left(\delta^{\prime} / x\right), x \rightarrow 0,
\end{aligned}
$$

for some constant $M_{1}$. The relation (2.12) indicates that it is no loss of generality to assume that $L(t)$ is nondecreasing. Hence,

$$
\left|I_{3}\right|<\varepsilon M_{1} x^{2 n+2} L(1 / x), x \rightarrow 0 .
$$

Finally, for some constant $M_{2}$,

$$
\begin{aligned}
\left|I_{4}\right| & \leq M_{2} x \int_{\delta^{\prime} / x}^{1 / x}(x t)^{2 n+1} g(t) d t \\
& =M_{2} x^{2 n+2} \int_{\delta^{\prime} / x}^{1 / x} t^{2 n+1} g(t) d t .
\end{aligned}
$$

By (2.12),

$$
\int_{\delta^{\prime} / x}^{1 / x} t^{2 n+1} g(t) d t=0\{L(1 / x)\}, x \rightarrow 0 .
$$

Hence, 


$$
I_{4}=o\left\{x^{2 n+2} L(1 / x)\right\}, x \rightarrow 0 .
$$

This completes the proof of the assertion

$$
I_{j}=0\left\{x^{2 n+2} L(1 / x)\right\}, \quad x \rightarrow 0, j=1,2 .
$$

It follows that

$$
G(x) \sim \sum_{r=1}^{n} a_{r} x^{2 r} \int_{0}^{\infty} t^{2 r-1} g(t) d t+a_{n+1} x^{2 n+2} \int_{0}^{1 / x} t^{2 n+1} g(t) d t, x \rightarrow 0 .
$$

Comparing this asymptotic relation with (2.10), we obtain (2.13).

REMARK 1. The assumption (2.9) is not necessary. With the help of some known results [9], [10], it can be shown that (2.10) itself implies $g(t)=0\left(t^{-\alpha_{1}}\right)$ as $t \rightarrow \infty$, for some. $\alpha_{1}>0$. This is sufficient to justify the Mellin transform technique used.

REMARK 2. The technique is quite general. In particular, it is applicable when $K(s)$, the Mellin transform of the kernel $k(t)$, has no singularities other than poles in the complex $s$-plane and, for $\sigma_{1} \leq 0 \leq \sigma_{2}, K(s)=0\left(|\tau|^{p}\right),|\tau| \rightarrow \infty, \quad s=\sigma+i \tau$.

\section{References}

[1] Dušan D. Adamović, "Sur quelques propriétés des fonctions à croissance lente de Karamata. I", Mat. Vesnik 3 (18) (1966), 123-136.

[2] Dušan D. Adamović, "Sur quelques propriétés des fonctions à croissance lente de Karamata. II", Mat. Vesnik 3 (18) (1966), 161-172.

[3] S. Aljančić, R. Bojanić et M. Tomić, "Sur la valeur asymptotique d'une classe des intégrales definies", Acad. Serbe Sci. Publ. Inst. Math. 7 (1954), 81-94.

[4] N.H. Bingham, "A Tauberian theorem for integral transforms of Hankel type", J. London Math. Soc. (2) 5 (1972), 493-503. 
[5] R. Bojanic and J. Karamata, "On slowly varying functions and asymptotic relations", MRC Technical Report No. 432 (University of Wisconsin, Madison, 1963).

[6] Arthur Erdélyi, Wi lhelm Magnus, Fritz Oberhettinger, Francesco G. Tricomi (edited by), Higher transcendental functions, Volume II. Based, in part, on notes left by Harry Bateman. (McGraw-Hill, New York, Toronto, London, 1953.)

[7] Arthur Erdélyi, Wilhelm Magnus, Fritz Oberhettinger, Francesco G. Tricomi (edited by), Tables of integral transforms, Volume I. Based, in part, on notes left by Harry Bateman. (McGraw-Hill, New York, Toronto, London, 1954.)

[8] E.J.G. Pitman, "On the behaviour of the characteristic function of a probability distribution in the neighbourhood of the origin", $J$. Austral. Math. Soc. 8 (1968), $423-443$.

[.9] K. Soni and R.P. Soni, "Lipschitz behavior and characteristic functions", SIAM J. Math. AnaZ. 4 (1973), 302-308.

[10] K. Soni and R.P. Soni, "Integrability theorems for a class of integral transforms", J. Math. Anal. Appl. 43 (1973), 397-418.

[11] K. Soni and R.P. Soni, "A note on probability distribution functions", submitted.

[12] E.C. Titchmarsh, The theory of functions, and ed. (Clarendon Press, Oxford, 1939).

[13] E.C. Titchmarsh, Introduction to the theory of Fourier integrals 2nd ed. (Clarendon Press, Oxford, 1948).

Department of Mathematics, University of Tennessee,

Knoxville, Tennessee, USA. 